\title{
ON THE APOSTERIORI ERROR ESTIMATES FOR FINITE-ELEMENT SOLUTIONS
}

\author{
RAIMONDAS ČIEGIS \\ Institute of Mathematics and Informatics \\ Vilnius Gediminas Technical University \\ Akademijos 4, LT-2600 Vilnius, Lithuania \\ E-mail: rc@fm.vtu.lt
}

\begin{abstract}
The analysis of the accuracy of the aposteriori error estimation procedure for finite-element solutions is presented. The function $Y-y$ is used as an aposteriori error estimator, here $y \in S_{0}^{1, \Delta}$ is the finite-element solution of the given problem and $Y \in S_{0}^{2, \Delta}$ is the high order solution of the same problem. The second order accuracy is proved for this error estimator in the $L_{2}, H_{1}$ and $L_{\infty}$ norms. Results of numerical experiments are presented.
\end{abstract}

\section{INTRODUCTION}

Numerical methods which are used in scientific computations and mathematical modelling must be robust and efficient. Both of these properties depend essentially on the quality of aposteriori error estimators. Firstly, similar to physical experiments, it is not sufficient to find a discrete solution, we also need to know the boundaries of the error of the obtained discrete solution. The key ingredient of such methodology is a reliable method for assesing the quality of computed approximation. An aposteriori error estimator must be computed using the data for the given problem and the discrete approximation itself. Such method is efficient if the costs of obtaining the estimator are small compared with the computation of the discrete solution [2]. Secondly, efficient numerical algorithms use adaptive approximations, which again depend on the quality of aposteriori error estimation procedures.

Aposteriori error estimates were investigated in many papers, see $[2 ; 5 ; 6]$. Mostly, the estimators are of residual type and are similar to estimators of Babuška, Rheinboldt [2]. The solution of only local problems on each element is used to get the error estimation. 
We consider the global error estimators, which are based on the high order finite-element solution of the given differential problem. Optimal accuracy estimates are proved for such aposteriori error estimators in the $L_{2}, L_{\infty}$ and $H_{1}$ norms.

The rest of the paper is outlined as follows. In section 2 we describe an elliptic problem and its discretization. In section 3 we construct the aposteriori error estimator and present the standard interpolation error estimates. The accuracy of the aposteriori error estimators is investigated in the $L_{2}$ norm in section 4. A similar analysis in the $H_{1}$ and $L_{i n f t y}$ norms is presented in section 5 and section 6 . Finally, in section 7 , numerical results are presented.

\section{MODEL PROBLEM AND FINITE ELEMENT APPROXIMA- TION}

We restrict the analysis to a simple model problem. Let consider the equation

$$
L u \equiv-\frac{d}{d x}\left(k(x) \frac{d u}{d x}\right)+q(x) u=f(x), \quad x \in(0,1)
$$

together with the boundary conditions

$$
u(0)=0, \quad u(1)=0 .
$$

The weak solution of the problem is given by

$$
(L u, v)=(f, v) \text { for all } v \in H_{0}^{1} .
$$

The Sobolev space $H_{0}^{1}$ consists of functions having square integrable first derivatives and vanishing at the boundary.

We introduce a partition

$$
\Delta=\left\{0=x_{0}<x_{1}<\ldots<x_{N}=1\right\}
$$

of the segment $[0,1]$ into $N$ subintervals and approximate $H_{0}^{1}$ by a finitedimensional subspace $S_{0}^{p, \Delta}$ of piecewice $p$-degree polynomials with respect to $\Delta$. The finite element solution $y \in S_{0}^{1, \Delta}$ is defined by the linear system

$$
(L y, v)=(f, v) \text { for all } v \in S_{0}^{1, \Delta} .
$$

Our aim is to study the efficiency of error estimators for the particular case of $p=1$, when most superconvergence estimates are degenerated. In order to simplify the details of the analysis we assume that the mesh $\Delta$ is uniform with the meshsize $h$. 


\section{APOSTERIORI ERROR ESTIMATORS}

Let consider the second finite element solution $Y \in S_{0}^{2, \Delta}$ which is defined by

$$
(L Y, v)=(f, v) \text { for all } v \in S_{0}^{2, \Delta} .
$$

We find it in the form [5]

$$
Y(x)=\sum_{j=1}^{N-1} Y_{j} \Phi_{j}(x)+\sum_{j=1}^{N} C_{j-0.5} \Psi_{j-0.5}(x),
$$

where $\Phi_{j}(x), \Psi_{j-0.5}(x)$ form a hierarchical basis for $S_{0}^{2, \Delta}$ and are given by

$$
\begin{gathered}
\Phi_{j}= \begin{cases}\left(x-x_{j-1}\right) /\left(x_{j}-x_{j-1}\right), & x_{j-1} \leq x \leq x_{j}, \\
\left(x_{j+1}-x\right) /\left(x_{j+1}-x_{j}\right), & x_{j} \leq x \leq x_{j+1}, \\
0 & \text { otherwise }\end{cases} \\
\Psi_{j-0.5}= \begin{cases}\left(x_{j}-x\right)\left(x-x_{j-1}\right) /\left(x_{j}-x_{j-1}\right)^{2}, & x_{j-1} \leq x \leq x_{j}, \\
0 & \text { otherwise. }\end{cases}
\end{gathered}
$$

Let denote the global error of the finite element solution as

$$
z(x)=u(x)-y(x), \quad z \in H_{0}^{1} .
$$

This error can be estimated by using the high order finite element solution $Y(x)$ (see $[5 ; 6]$ ). Let consider the aposteriori error estimator $Z=Y-y$. It satisfies the following problem

$$
(L Z, v)=(f, v)-(L y, v) \quad \text { for all } v \in S_{0}^{2, \Delta} .
$$

We note that the problem (5) is global and it involves the solution of a global elliptic problem.

The effectivity index

$$
\Theta_{l}=\frac{\|Z\|_{l}}{\|z\|_{l}}, \quad l=L_{2}, L_{\infty}, H_{1}
$$

is used to investigate the quality of error estimators $[2 ; 5 ; 6]$.The aposteriori error estimate $\hat{Z}$ is asymptotically exact, if

$$
\lim _{h \rightarrow 0} \frac{\|Z\|_{l}}{\|z\|_{l}}=1
$$


The order of accuracy of the apposteriori error estimate $Z$ is $\alpha$, if we have the following equality

$$
\|Z\|_{l}=\|z\|_{l}\left(1+O\left(h^{\alpha}\right)\right) .
$$

Theoretical analysis of aposteriori error estimators relies on standard interpolation estimates (see $[6 ; 8]$ )

$$
\begin{aligned}
\|z\|_{l} & \leq C(u) h^{p+1} \quad l=L_{2}, L_{\infty}, \\
\|z\|_{H_{1}} & \leq C(u) h^{p} .
\end{aligned}
$$

Estimates (6) guaranty that the aposteriori error estimator $Z$ is asymptotically exact with the first order of accuracy. The following inequalities follow from (6) :

$$
\begin{aligned}
\|y-u\| & \leq C h^{2}, \quad\|Y-u\| \leq C h^{3} \\
\|y-u\|_{L_{\infty}} & \leq C h^{2}, \quad\|Y-u\|_{L_{\infty}} \leq C h^{3} \\
\|y-u\|_{H_{1}} & \leq C h, \quad\|Y-u\|_{H_{1}} \leq C h^{2}
\end{aligned}
$$

Firstly we rewrite $Z$ as

$$
Z=Y-u+z .
$$

By using (7) we get the estimates

$$
\begin{aligned}
\|Z\|_{l} & \leq\|z\|_{l}\left(1+\frac{\|Y-u\|_{l}}{\|z\|_{l}}\right) \\
& =\|z\|_{l}(1+O(h)), \quad l=L_{2}, L_{\infty}, H_{1} . \\
\|Z\|_{l} & \geq\|z\|_{l}-\|Y-u\|_{l} \\
& =\|z\|_{l}(1-O(h)) .
\end{aligned}
$$

Hence we have proved that $\|Z\|_{l}$ is an asymptotically exact error estmator. The main problem is to investigate the order of accuracy of this estimator. It follows from (8) that $\alpha \geq 1$. In the remaining part of the paper we will prove that $\alpha=2$ for the error estimator $\|Z\|_{l}$.

\section{THE ACCURACY ANALYSIS IN THE $L_{2}$ NORM}

Firstly we define the interpolation polynomial $P_{2} u \in S_{0}^{2, \Delta}$, it satisfies the equalities

$$
\begin{array}{r}
\left(P_{2} u\right)\left(x_{j}\right)=u\left(x_{j}\right) \quad x_{j} \in \Delta, \\
\left(P_{2} u\right)\left(x_{j-0.5}\right)=u\left(x_{j-0.5}\right) .
\end{array}
$$


The explicit formula for $P_{2} u$ in the element $\left[x_{i}, x_{i+1}\right]$ is given by

$$
\begin{aligned}
P_{2} u & =u_{i} \frac{x_{i+1}-x}{h}+u_{i+1} \frac{x-x_{i}}{h} \\
& -2 \frac{u_{i+1}-2 u_{i+0.5}+u_{i}}{h^{2}}\left(x_{i+1}-x\right)\left(x-x_{i}\right) .
\end{aligned}
$$

We also will use the fact that spatial errors of $Y$ superconverge at nodes $x_{j} \in \Delta$

$$
\left|Y\left(x_{j}\right)-u\left(x_{j}\right)\right| \leq C h^{4}
$$

and at $x_{j-0.5}$

$$
\left|Y\left(x_{j-0.5}\right)-u\left(x_{j-0.5}\right)\right| \leq C h^{4}
$$

LEMMA 1. The high order finite-element solution $Y$ superconverges to the interpolation polynomial $P_{2} u$ and the approximation error is estimated by

$$
\left\|Y-P_{2} u\right\|_{L_{\infty}} \leq C h^{4}
$$

Proof. Let denote $v_{i}=Y_{i}-u_{i}$. Then it follows from (9) that

$$
\begin{aligned}
Y-P_{2} u & =v_{i} \frac{x_{i+1}-x}{h}+v_{i+1} \frac{x-x_{i}}{h} \\
& -2\left(v_{i+1}-2 v_{i+0.5}+v_{i}\right)\left(\frac{x_{i+1}-x}{h}\right)\left(\frac{x-x_{i}}{h}\right) .
\end{aligned}
$$

Then the statement of the lemma follows from the error estimates (10).

The function $Z$ can be rewritten as

$$
Z=z+P_{2} u-u+Y-P_{2} u \text {. }
$$

Let assume that $\left\|u^{(4)}\right\|_{L_{\infty}} \leq C_{4}$. Then using the Taylor expansion of the solution $u$ we obtain that the interpolation error of the polynomial $P_{2} u$ is given by

$$
P_{2} u-u(x)=\frac{1}{6} u^{\prime \prime \prime}\left(x_{i+0.5}\right)\left(x-x_{i}\right)\left(x-x_{i+0.5}\right)\left(x_{i+1}-x\right)+O\left(h^{4}\right) .
$$

We integrate $Z^{2}$ over the element $\left[x_{i}, x_{i+1}\right]$, use the estimates $(7),(11),(12)$ and obtain the equality

$$
\begin{array}{r}
\int_{x_{i}}^{x_{i+1}} Z^{2} d x=\int_{x_{i}}^{x_{i+1}} z^{2} d x+\frac{1}{3} u^{\prime \prime \prime} \\
\int_{x_{i}}^{x_{i+1}} z(x)\left(x-x_{i}\right)\left(x-x_{i+0.5}\right)\left(x_{i+1}-x\right) d x+O\left(h^{7}\right) .
\end{array}
$$


Let define the interpolation polynomial $P_{1} u$

$$
P_{1} u=u_{i} \frac{x_{i+1}-x}{h}+u_{i+1} \frac{x-x_{i}}{h} .
$$

It follows from the interpolation theory that

$$
P_{1} u-u(x)=-\frac{1}{2} u^{\prime \prime}\left(x_{i+0.5}\right)\left(x-x_{i}\right)\left(x_{i+1}-x\right)+O\left(h^{3}\right) .
$$

Then the error function $z$ can be rewritten as

$$
z(x)=z_{i} \Phi_{i}(x)+z_{i+1} \Phi_{i+1}-\frac{1}{2} u^{\prime \prime}\left(x_{i+0.5}\right)\left(x-x_{i}\right)\left(x_{i+1}-x\right)+O\left(h^{3}\right) .
$$

We need to estimate three integrals in (13). After simple calculations we get

$$
\begin{aligned}
\int_{x_{i}}^{x_{i+1}}\left(x-x_{i}\right)^{2}\left(x-x_{i+0.5}\right)\left(x_{i+1}-x\right)^{2} d x & =0 \\
\int_{x_{i}}^{x_{i+1}}\left(x-x_{i}\right)\left(x-x_{i+0.5}\right)\left(x_{i+1}-x\right)^{2} d x & =-\frac{h^{5}}{120} \\
\int_{x_{i}}^{x_{i+1}}\left(x-x_{i}\right)^{2}\left(x-x_{i+0.5}\right)\left(x_{i+1}-x\right) d x & =\frac{h^{5}}{120} .
\end{aligned}
$$

Then it follows from (13)

$$
\int_{x_{i}}^{x_{i+1}} Z^{2} d x=\int_{x_{i}}^{x_{i+1}} z^{2} d x+\frac{h^{5}}{360} u^{\prime \prime \prime}\left(x_{i+0.5}\right) \frac{z_{i+1}-z_{i}}{h}+O\left(h^{7}\right) .
$$

We use the well-known notation of finite-differences

$$
y_{x}=\frac{y_{i+1}-y_{i}}{h}, \quad y_{\bar{x}}=\frac{y_{i}-y_{i-1}}{h} .
$$

Lemma 2. The finite differences $z_{x}$ superconverge at grid nodes :

$$
\left|\frac{z_{i+1}-z_{i}}{h}\right| \leq C h^{2}, \quad i=1,2, \cdots, N-1
$$

Proof. The vector $z$ satisfies the following finite difference problem (see [7])

$$
\begin{array}{r}
-\left(a z_{\bar{x}}\right)_{x}+d_{i} z_{i}=\eta_{x}+\mu, \\
z_{0}=0, \quad z_{N}=0,
\end{array}
$$

where the coefficients $a$ and $d$ satisfy the conditions

$$
a_{i} \geq k_{0}>0, \quad d_{i} \geq 0
$$


and the truncation errors $\eta$ and $\mu$ are estimated by

$$
\left|\eta_{i}\right| \leq C h^{2}, \quad\left|\mu_{i}\right| \leq C h^{2} \quad \text { for all } x_{i} \in \Delta
$$

Since we have from (7) that

$$
\left|z_{i}\right| \leq C h^{2} \quad \text { for all } x_{i} \in \Delta
$$

then it is sufficient to consider the problem

$$
\begin{array}{r}
-\left(a z_{\bar{x}}\right)_{x}=\eta_{x}+\mu, \\
z_{0}=0, \quad z_{N}=0 .
\end{array}
$$

After simple calculations we obtain the solution $z$ in the explicit form

$$
\begin{aligned}
z_{\bar{x}} & =\frac{1}{a_{i-0.5}}\left(C-\eta_{i-0.5}-\sum_{j=1}^{i-1} \mu_{j} h\right) \\
C & =\sum_{i=1}^{N} \frac{h}{a_{i-0.5}}\left(\eta_{i-0.5}+\sum_{j=1}^{i-1} \mu_{j} h\right) / \sum_{i=1}^{N} \frac{1}{a_{i-0.5}}
\end{aligned}
$$

The lemma is proved.

Summing integral equalities (15) over $i$ yields

$$
\int_{0}^{1} Z^{2} d x=\int_{0}^{1} z^{2} d x+O\left(h^{6}\right) .
$$

The global error $z$ satisfies the inequality (7), so we have the estimate

$$
\|Z\|_{L_{2}}^{2}=\|z\|_{L_{2}}^{2}\left(1+O\left(h^{2}\right)\right)
$$

or

$$
\|Z\|_{L_{2}}=\|z\|_{L_{2}}\left(1+O\left(h^{2}\right)\right) .
$$

Hence we have proved that the aposteriori error estimator $\|Z\|_{L_{2}}$ has the second order of the accuracy.

\section{THE ACCURACY ANALYSIS IN THE $H_{1}$ NORM}

The function $Z^{\prime}$ can be rewritten as

$$
Z^{\prime}=z^{\prime}+\left[\left(P_{2} u\right)^{\prime}-u^{\prime}\right]+\left[Y^{\prime}-\left(P_{2} u\right)^{\prime}\right]
$$


Lemma 3. The high order finite-element solution $Y^{\prime}$ superconverges to the interpolation polynomial $\left(P_{2} u\right)^{\prime}$ and the approximation error is estimated by

$$
\left|Y^{\prime}-\left(P_{2} u\right)^{\prime}\right| \leq C h^{3} .
$$

Proof. Let denote $v_{i}=Y_{i}-u_{i}$. Then it follows from (9) that

$$
Y^{\prime}-\left(P_{2} u\right)^{\prime}=\frac{v_{i+1}-v_{i}}{h}+4\left(v_{i+1}-2 v_{i+0.5}+v_{i}\right)\left(\frac{x_{i+0.5}-x}{h^{2}}\right)
$$

Then the statement of the lemma follows from the superconvergence error estimates (10).

The first derivative of the global error function $z$ can be represented as

$$
z^{\prime}(x)=z_{x}+u^{\prime \prime}\left(x_{i+0.5}\right)\left(x-x_{i+0.5}\right)+O\left(h^{2}\right) .
$$

The interpolation error of the polynomial $\left(P_{2} u\right)^{\prime}$ is given by ( see (12))

$$
\left(P_{2} u\right)^{\prime}-u^{\prime}(x)=\frac{1}{6} u^{\prime \prime \prime}\left(x_{i+0.5}\right)\left[\left(x-x_{i}\right)\left(x_{i+1}-x\right)+2\left(x-x_{i+0.5}\right)^{2}\right]+O\left(h^{3}\right) .
$$

We integrate $\left(Z^{\prime}\right)^{2}$ over the element $\left[x_{i}, x_{i+1}\right]$, use the estimates $(7),(19),(20)$ and obtain the equality

$$
\begin{aligned}
\int_{x_{i}}^{x_{i+1}}\left(Z^{\prime}\right)^{2} d x & =\int_{x_{i}}^{x_{i+1}}\left(z^{\prime}\right)^{2} d x \\
& +\frac{1}{3} u^{\prime \prime \prime}\left(x_{i+0.5}\right) \int_{x_{i}}^{x_{i+1}} z_{x}\left[\left(x-x_{i+0.5}\right)^{3}\right. \\
& \left.+\left(x-x_{i}\right)\left(x-x_{i+0.5}\right)\left(x_{i+1}-x\right)\right] d x+O\left(h^{4}\right) .
\end{aligned}
$$

After simple calculations we get

$$
\begin{aligned}
\int_{x_{i}}^{x_{i+1}}\left(x-x_{i+0.5}\right)^{3} d x & =0, \\
\int_{x_{i}}^{x_{i+1}}\left(x-x_{i}\right)\left(x-x_{i+0.5}\right)\left(x_{i+1}-x\right) d x & =0 .
\end{aligned}
$$

Summing integral equalities (21) over $i$ yields

$$
\int_{0}^{1}\left(Z^{\prime}\right)^{2} d x=\int_{0}^{1}\left(z^{\prime}\right)^{2} d x+O\left(h^{4}\right)
$$

The global error $z^{\prime}$ satisfies the inequality (7), so we have the estimate

$$
\|Z\|_{H_{1}}^{2}=\|z\|_{H_{1}}^{2}\left(1+O\left(h^{2}\right)\right)
$$


or

$$
\|Z\|_{H_{1}}=\|z\|_{H_{1}}\left(1+O\left(h^{2}\right)\right) .
$$

Hence we have proved that the aposteriori error estimator $\|Z\|_{H_{1}}$ has the second order of the accuracy.

\section{THE ACCURACY ANALYSIS IN THE $L_{\infty}$ NORM}

It follows from previous sections that the function $Z$ can be rewritten as

$$
Z=z+P_{2} u-u+Y-P_{2} u
$$

We have proved in Lemma 1 that

$$
\left|Y(x)-\left(P_{2} u\right)(x)\right| \leq C h^{4}
$$

The interpolation error of the polynomial $P_{2} u$ is given by (12):

$$
P_{2} u-u(x)=\frac{1}{6} u^{\prime \prime \prime}\left(x_{i+0.5}\right)\left(x-x_{i}\right)\left(x-x_{i+0.5}\right)\left(x_{i+1}-x\right)+O\left(h^{4}\right) .
$$

Let assume that

$$
\|z\|_{L_{\infty}}=|z(\tilde{x})| \quad x_{i} \leq \tilde{x} \leq x_{i+1}
$$

Lemma 4. The point $\tilde{x}$ satisfies the following equality

$$
\tilde{x}=x_{i+0.5}+C h^{2} .
$$

Proof. The point $\tilde{x}$ is determined from the equation

$$
z^{\prime}(x)=0
$$

The global error function $z$ can be rewritten as

$$
z=y-P_{1} u+P_{1} u-P_{2} u+P_{2} u-u,
$$

hence we have the equation

$$
z_{x}-4 u_{\bar{x} x}\left(x-x_{i+0.5}\right)+\left(P_{2} u\right)^{\prime}-u^{\prime}=0 .
$$

It follows from Lemma 2 and from the interpolation theory that

$$
\left|z_{x}\right| \leq C h^{2}, \quad\left|\left(P_{2} u\right)^{\prime}(x)-u^{\prime}(x)\right| \leq C h^{2},
$$


so the equality (23) is valid if $\left|u_{\bar{x} x}\right|>0$.

We get from Lemma 1 and equalities (12), (23) that

$$
Z(\tilde{x})=z(\tilde{x})+O\left(h^{4}\right) .
$$

Let assume that

$$
\|Z\|_{L_{\infty}}=|Z(\hat{x})|
$$

Lemma 5. The point $\hat{x}$ satisfies the following equality

$$
\hat{x}=x_{i+0.5}+C h^{2} .
$$

Proof. The point $\hat{x}$ is determined from the eqaution

$$
Z^{\prime}(x)=0 .
$$

The global error function $Z$ can be rewritten as

$$
Z=y-P_{1} u+P_{1} u-P_{2} u+P_{2} u-Y,
$$

hence we have the equation

$$
z_{x}-4 u_{\bar{x} x}\left(x-x_{i+0.5}\right)+\left(P_{2} u\right)^{\prime}-Y^{\prime}=0 .
$$

Now it is sufficient to use the results of Lemma 2 and Lemma 3.

It follows from (22) that

$$
Z(x)=z(x)+C h^{4}, \quad \text { for any } x=x_{i+0.5}+C H^{2} .
$$

Using (22),(25) and (26) yields

$$
\|z\|_{L_{\infty}}-O\left(h^{4}\right) \leq\|Z\|_{L_{\infty}} \leq\|z\|_{L_{\infty}}+O\left(h^{4}\right)
$$

or

$$
\|Z\|_{L_{\infty}}=\|z\|_{L_{\infty}}\left(1+O\left(h^{2}\right)\right) .
$$

Hence we have proved that the aposteriori error estimator $\|Z\|_{L_{\infty}}$ has the second order of the accuracy. 
Table 1.

Converges rates of the aposteriori error estimators

\begin{tabular}{lcccc}
\hline$N$ & $L_{2}$ & $L_{\infty}$ & $L_{\infty, h}$ & $H_{1}$ \\
\hline 80 & 1.096 & 1.065 & 0.938 & 1.209 \\
160 & 1.511 & 1.371 & 1.585 & 1.606 \\
320 & 1.802 & 1.650 & 1.722 & 1.854 \\
640 & 1.941 & 1.816 & 1.953 & 1.957 \\
\hline
\end{tabular}

\section{NUMERICAL RESULTS}

Let consider the problem (1) with the following coefficients (see [2]):

$$
k(x)=1, \quad q(x)=x+0.01, \quad r=-0.25, \quad \alpha=0.01 .
$$

The function $f$ and boundary conditions are chosen so that the exact solution of this problem is the function

$$
u(x)=(x+\alpha)^{r}-\left[\alpha^{r}(1-x)+(1+\alpha)^{r} x\right] .
$$

We solved the problem on uniform spatial meshes having $N=80,160$, 320 , and 640 elements and on various types of nonuniform meshes, including asymptotically optimal meshes (see [2]). Similar results were obtained in all cases.

Table 1 shows the values of effectivity indices $\Theta_{l}$ corresponding to the error estimators in the $L_{2}, L_{\infty}, H_{1}$ norms. $L_{\infty, h}$ denotes the discrete pointwise maximum norm at mesh nodes. More results of numerical experiments are given in [3].

It is proved that aposteriori error estimates for elliptic problems converge to the true error with the second order of the accuracy. Computational results indicate that the asymptotic order of the accuracy is achieved for relatively small numbers of elements $N$.

\section{REFERENCES}

[1] M. Ainsworth, The influence and selection of subspaces for aposteriori error estimators, Numer. Math., 73 (1996), 399-418.

[2] I. Babuska, W. Rheinboldt, Analysis of optimal finite-element meshes in $R^{1}$, Math. of Comp., 33 (1979), 435-463.

[3] R. Čiegis, On the accuracy of aposteriori estimates for finite for finite element schemes, Pitman Research Notes in Mathematics, Series 375, Integral methods in science and engineering, 1997, 74-78.

[4] J. Hugger, An asymptotically exact, pointwise, aposteriori error estimator for the finite element method with super convergence properties, Adaptive methods for PDE, 1993, $277-305$. 
[5] P. Moore, J. Flaherty, A local refinement finite element method for one-dimensional parabolic systems, SIAM J. Numer. Anal., 27 (1990), 1422-1444.

[6] P. Moore, A posteriori error estimation with finite element semi- and fully discrete methods for nonlinear parabolic equations in one space dimension, SIAM J. Numer. Anal., 31 (1994), 149-169.

[7] A.A.Samarskii, The theory of difference schemes, Nauka, Moscow, 1978,(in Russian ).

[8] V.Thomee, Galerkin finite element methods for parabolic problems, Springer - Verlag, Berlin, 1984. 